\title{
Preface
}

\section{Medications and Their Effects on Sleep and Wake}

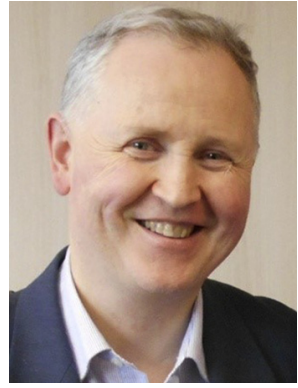

Johan Verbraecken, MD, PhD

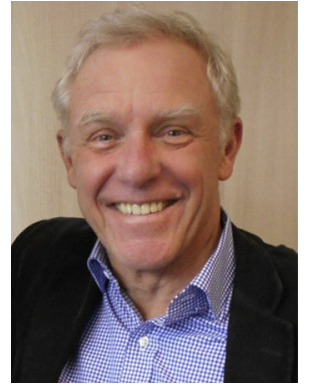

Jan Hedner, MD, PhD

Editors

Welcome to this issue of Sleep Medicine Clinics, which is dedicated to the topic of medications and their effects on sleep/wake mechanisms. This is a comprehensive overview of current drug therapy in different sleep disorders and of the complex relationship between drugs and sleep-wake.

The number of patients suffering from sleeprelated disorders is increasing dramatically, and drug prescription is a common therapeutic measure. Several effective pharmaceuticals have been developed and launched only during the last decade. The sleep disorders addressed in this issue are diverse, but reflect those most relevant with respect to disorders of sleep. Invited authors are experts in their field and represent recognized institutions from all over the world. The introductory article offers a comprehensive overview of the neurochemistry of sleep and wake. Dr Sebastian Holst, Copenhagen University, Denmark, and Prof Hans-Peter Landolt, University of Zürich, Switzerland, are preeminent experts in the area and provide the reader with an up-todate overview. The following articles in this issue are essentially based on these neurochemistry insights. In another article in this issue, which focused on sleep-related side effects of drugs, Dr Ann Van Gastel, from the Antwerp University Hospital, Belgium, brings two decades of research experience to delineate and discuss this topic. Subsequently, Prof Ludger Grote, from the Gothenburg University, Sweden, addresses sleepdisordered breathing and ventilatory impairment, which occur as a consequence of drug use. The following parts of this issue deal with the potential pharmacologic therapies to consider in the major sleep disorders encountered in clinical sleep medicine. First, we explore the pharmacologic treatment of the most common sleep-related complaint, insomnia. Dr Sylvie Dujardin and colleagues, from the Centre for Sleep Medicine Kempenhaeghe, Heeze, The Netherlands, provide a review of the current pharmacologic management means. Next, Prof Gert Jan Lammers from the University of Leiden and Sleep Centre, SEIN, The Netherlands, provides a cutting-edge update on the current pharmacologic approach in narcolepsy and hypersomnia, and Dr Paola Proserpio and colleagues, from Milan, Pavia, Italy, discuss the current treatment of parasomnias, long considered to be disorders of primarily emotional origin, in their review. Prof Jan Hedner, co-editor, and Dr Ding Zou, from Gothenburg University, Sweden, provide an exhaustive review of what is known about the pharmacologic management of sleep-disordered breathing, and where we might hope to go in the coming decade. Next follows a review of drug therapy in restless legs syndrome by $\mathrm{Dr}$ Galia Anguelova and colleagues from The Hague, The Netherlands, and a current update on drugs used in circadian sleep-wake rhythm disorders as well a detailed description of the role of light and melatonin in these disorders by Prof Helen Burgess, from Chicago, Illinois, and Prof Jonathan Emens, from Portland, Oregon.

Before turning to the final two articles, we have addressed special issues that follow with 
medication in the specific populations of pregnant women and children. Pregnancy is covered by Prof Laura McLafferty, from Philadelphia and Dr Meredith Spada and Dr Priya Gopalan, from Pittsburgh, Pennsylvania, while the pediatric aspects are summarized by Prof Paschalis Steiropoulos, from Alexandroupolis, and $\mathrm{Dr}$ Nicholas-Tiberio Economou, from Athens, Greece, together with Prof Luigi Ferini-Strambi, from Milan, Italy. Finally, we conclude with two articles to remind the reader this topic is not merely one of prescribing drugs but also of their withdrawal. These articles address discontinuation of hypnotic drugs by $\mathrm{Dr}$ Jonathan Hintze and Prof Jack Edinger, from Denver, Colorado, and of chronic opioid medication by Prof Michelle Cao from Stanford University, California, and Prof Shahrokh Javaheri, from Cincinnati, Ohio, with respect to their influence on sleep. These areas are arguably some of the most complex, maligned, and infrequently studied topics in the field of sleep medicine. Frequently, there is a need of cognitive behavioral therapy and concerted multidisciplinary efforts to deal with these complex sleep disorders.

In summary, it is our hope that this issue of Sleep Medicine Clinics will provide the reader with a better understanding of both the usefulness and the limitations that follow therapeutic drug use in various sleep disorders. Ultimately, we anticipate that the information communicated in this issue will benefit our patients in their quest of better sleep and a better quality of life. We are indebted to the contributing authors of this issue for taking time out of their busy schedules to realize this series. Without them, the project would have been impossible. We also thank Donald Mumford at Elsevier for his tenacious efforts in pushing this project through to its final form.

Johan Verbraecken, MD, PhD Multidisciplinary Sleep Disorders Centre Antwerp University Hospital and University of Antwerp

Wilrijkstraat 10

Edegem, Antwerp 2650, Belgium

Jan Hedner, MD, PhD

Centre for Sleep and Wakefulness Disorders Sahlgrenska Academy University of Gothenburg Medicinaregatan 8B Gothenburg 41346, Sweden

E-mail addresses:

johan.verbraecken@uza.be (J. Verbraecken) Jan.hedner@lungall.gu.se (J. Hedner) 